\title{
Commentary: Incorporation of membrane-anchored flagellin or Escherichia coli heat-labile enterotoxin B subunit enhances the immunogenicity of rabies virus-like particles in mice and dogs
}

\author{
Arun Kumar * \\ T-cell Platform, Translational Medicine, Novartis Vaccines and Diagnostics (a GSK Company), Siena, Italy
}

Keywords: rabies, VLP, vaccines, recombinant proteins, viruses

\section{A commentary on}

OPEN ACCESS

Edited by:

Masayuki Saijo,

National Institute of Infectious

Diseases, Japan

Reviewed by:

Takashi Irie

Hiroshima University, Japan Akira Nishizono,

Oita University, Japan

${ }^{*}$ Correspondence:

Arun Kumar

arun.q.kumar@gsk.com; sharmakumar.arun@yahoo.com

Specialty section:

This article was submitted to Virology,

a section of the journal

Frontiers in Microbiology

Received: 10 June 2015 Accepted: 11 September 2015 Published: 29 September 2015

Citation:

Kumar A (2015) Commentary: Incorporation of membrane-anchored flagellin or Escherichia coli heat-labile enterotoxin $B$ subunit enhances the immunogenicity of rabies virus-like particles in mice and dogs.

Front. Microbiol. 6:1039

doi: 10.3389/fmicb.2015.01039
Incorporation of membrane-anchored flagellin or Escherichia coli heat-labile enterotoxin B subunit enhances the immunogenicity of rabies virus-like particles in mice and dogs by Qi, Y. (2015). Front. Microbiol. 6:169. doi: 10.3389/fmicb.2015.00169

Rabies virus is accountable for substantial economic burden, and possesses high risk to the individuals living in low-income countries. Rabies is fatal encephalitis caused by the rabies virus (RABV) and usually transmit to humans through bites from rabid animals. The causative agent for this life threatening disease is RABV, containing single-stranded negative-sense RNA as genetic material (Jackson, 2013). Annually, rabies is responsible for approximately 55,000 deaths worldwide (a substantial part of deaths occur in developing countries); (Ge et al., 2011). Vaccination is the principal path for prevention and control of the disease. Currently available cell culture based rabies vaccines possess several side effects (e.g. fever, headache, neurological disease etc.) and high cost in developing countries usually require many doses to provide pre and post-exposure protection against the RABV. Several, improved vaccines based on antirabies monoclonal antibodies, lyophilized, and cell culture technology, are already under clinical development phase (Kaur et al., 2015). Furthermore, recombinant molecules have also been used as vaccine candidates very extensively. Further, immunogenicity of these molecules can be enhanced by combining adjuvants and immuno-stimulatory molecules. In the past, Wen et al. demonstrated that recombinant rabies virus (rRABV) expressing dendritic cell activating molecules such as granulocyte-macrophage colony stimulating factor (GM-CSF), macrophage-derived chemokine (MDC), and macrophage inflammatory protein (MIP-1a), could enhance RABV immunogenicity (Wen et al., 2011). Moreover, Zhou et al. suggested that rRABV expressing bacterial flagellin could also induce robust adaptive immune responses (Zhou et al., 2013). Flagellin is a very effective and potent adjuvant because of non-inducer of IgE production and at very low dose $(1-10 \mu \mathrm{g}$ in nonhuman primates) very efficient to induce effective immune responses (Mizel and Bates, 2010). Recombinant virus-like particles (VLPs) are promising vaccine candidates, because they mimic original virus structure and are able to induce potent humoral and cellular immune responses (Kumar et al., 2011). Moreover, VLP possesses several advantages over the conventional vaccines because of the lack of genetic material, stable in extreme environment and capable of expressing foreign molecules. Very recently, Fontana et al. (2014) showed that VLPs containing glycoprotein of 
RABV are very efficient in induction of antibody mediated immune responses in Balb/c mice. Qi et al. (2015) combined all these novel strategies, in order to develop an effective RABV vaccine. In present report, Qi and colleagues constructed chimeric RABV-like particles (cRVLPs) containing RABV glycoprotein and matrix protein, expressing membraneanchored flagellin or E. coli heat-labile enterotoxin B (LTB). Immunological studies in $\mathrm{BALB} / \mathrm{c}$ mice and $\mathrm{dog}$ models demonstrated that both humoral and cellular immune responses were stronger with cRVLPs, compared with standard rabies VLPs (sVLPs). Further, Qi et al. (2015) suggest that flagellin promote antigen processing and presentation by inducing higher expression of MHC I and II on the surface of DCs. Interestingly,

\section{References}

Fontana, D., Kratje, R., Etcheverrigaray, M., and Prieto, C. (2014). Rabies virus-like particles expressed in HEK293 cells. Vaccine 32, 2799-2804. doi: 10.1016/j.vaccine.2014.02.031

Ge, J., Wang, X., Tao, L., Wen, Z., Feng, N., Yang, S., et al. (2011). Newcastle disease virus-vectored rabies vaccine is safe, highly immunogenic, and provides long-lasting protection in dogs and cats. J. Virol. 85, 8241-8252. doi: 10.1128/JVI.00519-11

Jackson, A. C. (2013). Current and future approaches to the therapy of human rabies. Antiviral Res. 99, 61-67. doi: 10.1016/j.antiviral.2013. 01.003

Kaur, M., Garg, R., Singh, S., and Bhatnagar, R. (2015). Rabies vaccines: where do we stand, where are we heading? Expert Rev. Vaccines 14, 369-381. doi: 10.1586/14760584.2015.973403

Kumar, A., Chen, T., Pakkanen, S., Kantele, A., Söderlund-Venermo, M., Hedman, K., et al. (2011). T-helper cell-mediated proliferation and cytokine responses against recombinant Merkel cell polyomavirus-like particles. PLoS ONE 6:e25751. doi: 10.1371/journal.pone.0025751

Mizel, S. B., and Bates, J. T. (2010). Flagellin as an adjuvant: cellular mechanisms and potential. J. Immunol. 185, 5677-5682. doi: 10.4049/jimmunol. 1002156

Qi, Y., Kang, H., Zheng, X., Wang, H., Gao, Y., Yang, S., et al. (2015). Incorporation of membrane-anchored flagellin or Escherichia coli heat-labile enterotoxin B subunit enhances the immunogenicity of rabies virus-like in a pioneer study, mucosal delivery of inactivated influenza vaccine have been shown to induce heterotypic immunity against H1N1 virus, when administered with heat-labile enterotoxin B (Tumpey et al., 2001). These attributes make flagellin and enterotoxin B outstanding adjuvants for use in vaccines.

In conclusion, present and previous reports suggest that chimeric VLPs are novel class of subunit vaccines and possess capability to induce both protective innate and adaptive immune responses. Possibly, use of immuno-modulators along with cRVLPS may enhance the efficacy of existing rabies vaccines. Further, detailed mechanism of the comprehensive effect of flagellin and enterotoxin B stimulated T and B-cells, on antiviral immune responses, should be investigated.

particles in mice and dogs. Front. Microbiol. 6:169. doi: 10.3389/fmicb.2015. 00169

Tumpey, T. M., Renshaw, M., Clements, J. D., and Katz, J. M. (2001). Mucosal delivery of inactivated influenza vaccine induces B-cell-dependent heterosubtypic cross-protection against lethal influenza A H5N1 virus infection. J. Virol. 75, 5141-5150. doi: 10.1128/JVI.75.11.5141-5150.2001

Wen, Y., Wang, H., Wu, H., Yang, F., Tripp, R. A., Hogan, R. J., et al. (2011). Rabies virus expressing dendritic cell-activating molecules enhances the innate and adaptive immune response to vaccination. J. Virol. 85, 1634-1644. doi: 10.1128/JVI.01552-10

Zhou, M., Zhang, G., Ren, G., Gnanadurai, C. W., Li, Z., Chai, Q., et al. (2013). Recombinant rabies viruses expressing GM-CSF or flagellin are effective vaccines for both intramuscular and oral immunizations. PLoS ONE 8:e63384. doi: 10.1371/journal.pone.0063384

Conflict of Interest Statement: The author declares that the research was conducted in the absence of any commercial or financial relationships that could be construed as a potential conflict of interest.

Copyright (C) 2015 Kumar. This is an open-access article distributed under the terms of the Creative Commons Attribution License (CC BY). The use, distribution or reproduction in other forums is permitted, provided the original author(s) or licensor are credited and that the original publication in this journal is cited, in accordance with accepted academic practice. No use, distribution or reproduction is permitted which does not comply with these terms. 